\title{
Simplified evaluation of a building impacted by a terrorist explosion
}

\author{
D. Makovička ${ }^{1} \&$ D. Makovička Jr. ${ }^{2}$ \\ ${ }^{1}$ Czech Technical University in Prague, Klokner Institute, Czech Republic \\ ${ }^{2}$ Static and Dynamic Consulting, Czech Republic
}

\begin{abstract}
This paper determines the parameters of the explosion wave excited by a charge planted by terrorists. A suitcase containing an industrial explosive (Danubit I, mass 6.45 kilogram) remotely controlled by mobile telephone was placed in the left-luggage office of a railway station. Certain simplified methods according to various publications, according to our own experimental results and according to 3D computations based on detailed calculation modelling of the interior of the room are compared to determine the explosive effects. Equivalent static analysis was applied to the dynamic response of the structural elements of the room (walls, floor, roof and windows). The damage caused to these structural elements is weighted on the basis of the angle of fracture of the central axis / surface, and on the basis of the limit stress state of these structures.
\end{abstract}

Keywords: explosion, room structure, simplified analysis, dynamic response, failure prognosis.

\section{Introduction}

When a small charge explodes in the internal space of a building, a pressure wave is formed by the explosion that applies a load on the surrounding internal elements of the structure (Figure 1). The pressure effects of even a small charge are usually high, and the primary consequence is that a window or a door structure may be broken and the pressure is released into the surrounding areas. Although the exhaust vents open, the load transmitted to the surrounding walls of the room, and to the ceiling and floors, is quite high, and the corresponding magnitude must first be estimated. This magnitude depends on numerous 


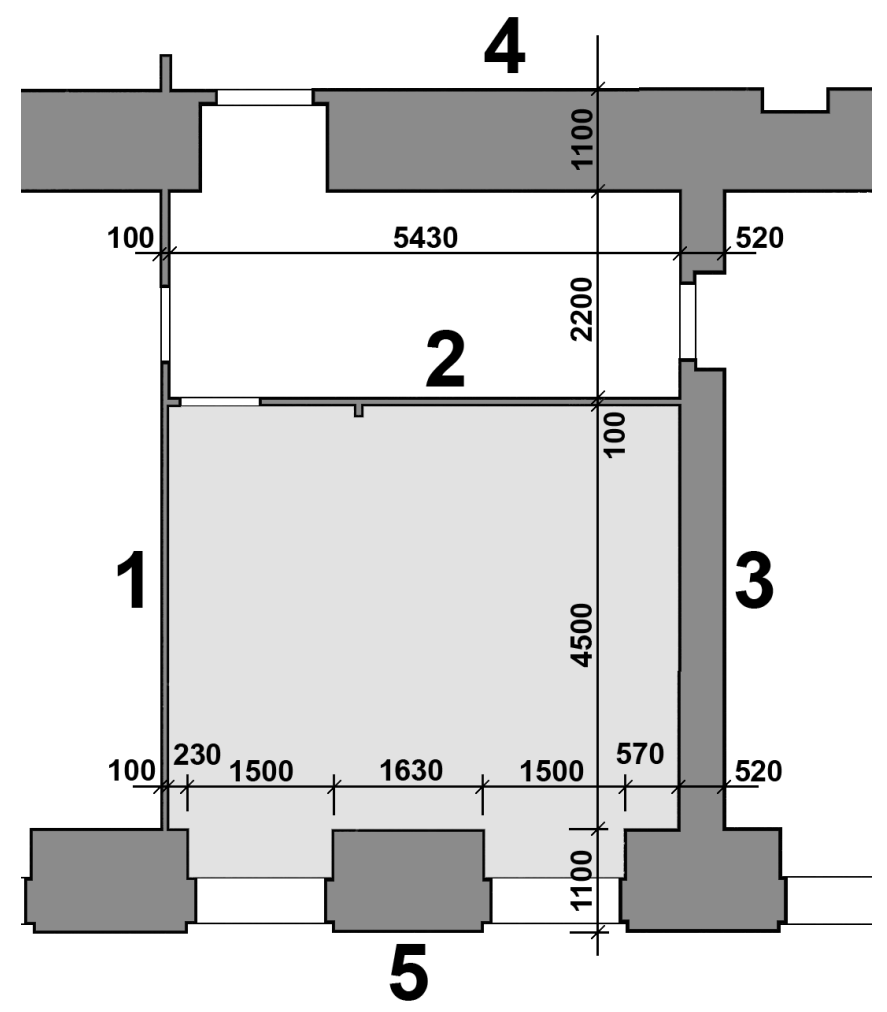

Figure 1: $\quad$ Ground plan of a room located on the ground floor of a building (the surrounding walls are numbered to provide easier identification of the response calculation).

parameters that have an impact on the load level, and therefore it is appropriate to adopt simplifying assumptions.

The load of the surrounding structure of the room can be determined either by means of relatively accurate calculations which take into account the internal space of the room, the composition of the explosive, and which deal with the interaction of the internal environment (air and combustion product mixture) with the structure of the room itself. Alternatively, simpler approximate procedures can be applied; these procedures are based on determining the parameters of the explosion load in a free space and then approximating them to the load in a semi-enclosed space (after the exhaust vents have opened).

\section{Behaviour of masonry when loaded by an explosion}

When dynamically loaded by an explosion, masonry displays almost linear elasticity up to the point of failure [6]. The real elastic modulus $E$ is an important 
material quantity for calculating the response of masonry to the load due to an explosion. It can be estimated according to the deformability modulus $E_{\text {def }}$ stated in the design standards, based on the experimentally verified experience of the authors of this paper:

a) To calculate the dynamic response of an undamaged structure near the failure limit:

$$
E \approx 0.5 \cdot E_{\text {def }}
$$

b) To calculate the dynamic response of a structure already damaged by visible cracks:

$$
E \approx 0.1 \cdot E_{\text {def }}
$$

The loading capacity of the brick masonry is decided in accordance with the standards used to design the bending compression / tensile strength of the masonry, with a certain margin of safety, given by the coefficients of the load, combination, etc.

If the loading capacity $R_{t f d}$ is exceeded, a crack appears in the structure of the material. Thus the most unfavourable condition must apply to a safe structure, based on comparing the stress state or the deformation magnitude. The following relationship applies to the stress combination:

$$
\min \left(\sigma_{g} \pm \sigma_{\text {expl }}\right) \geq-R_{t f d} \text { or upon adjustment } \sigma_{\text {expl }}-\sigma_{g} \leq R_{t f d}
$$

where $\sigma_{\text {expl }}$ is stress caused by the effects of the pressure wave when there is an explosion, $\sigma_{g}$ is the normal stress at a given place (a joint) caused by the overburden weight itself.

In structural design based on limit state theory, it is usually more suitable to consider the carrying capacity strength moment, possibly in combination with the normal force, rather than the carrying capacity limit $R_{t f d}$. This stress criterion must be supplemented by an evaluation of the deformation of the structure. As a rule, the limit deformation value (shift or angular displacement) determines the actual destruction of the wall; the limit deformation value corresponds to the critical angle of the partial turning of the central line of the structure due to its bending. The limit angular displacement $\psi$ at the failure limit is found in the range of approximately $2.3^{\circ}$ to $5.7^{\circ}$ for masonry $[6,9]$, a minimum of $6.5^{\circ}$ for reinforced concrete [3], and a minimum of $10.5^{\circ}$ for steel [3]:

$$
\psi=2 \operatorname{arctg}(2 y / l)
$$

where $y$ is the maximum achieved deflection of the board (at the centre of the span), and $l$ is the structure span along the shorter dimension.

\section{Explosion load}

When a charge explodes in an open space, the pressure effect of the impact wave on an obstacle (the load of the building structure) depends on the situation of the building with respect to the focus of the explosion, the impact wave parameters, etc. The entire phenomenon of the impact wave effect on the structure is then 
usually simplified for calculation purposes, using numerous assumptions, especially as regards the intensity and the time course of the impact wave effect and its distribution in contact with the given object $[2,7]$. When an actual event takes place, the specific course of the load action depends on the swirl flow bypassing the structure surface, the atmospheric pressure, the temperature conditions and other factors that are usually neglected in a simplified analysis. The parameters of the explosive, too, are determined on the basis of average values; empirical formulas are used, and operate with mean (probable) coefficient levels. Thus the structure calculations concerning the impact wave effects are significantly burdened by these inaccuracies in the input quantities of the entire phenomenon.

Empirical formulas created by various authors [1, 3, 4, 7] are usually used for the time course of the pressure wave and subsequently the structure load. The structure of the formulas according to various authors is very similar, and they usually differ only in the magnitudes of the coefficients. Due to the variability of these coefficients, the uncertainty of the formulas is usually found to be in the range of $\pm 20 \%$, and possibly even more. The reliability of individual formulas improves with increasing distance of the pressure wave from the focus of the explosion.

The overpressure determined at the face of the air impact wave that spreads from the explosion site to the surroundings stems from the reduced distance $[1,3,7,8]$ is:

$$
\bar{R}=\frac{R}{\sqrt[3]{C_{w}}}
$$

where $\bar{R}$ is the reduced separation distance from the epicentre of the explosion $\left[\mathrm{m} / \mathrm{kg}^{1 / 3}\right], R$ is the distance from the explosion epicentre $[\mathrm{m}]$, and $C_{W}$ is the equivalent mass of the charge [kg TNT].

It is assumed that the energy released by the explosion is proportional to the mass of the explosive, and the solution consists in introducing a reference charge chosen to be represented by tritol (trinitrotoluene, TNT). Therefore the mass of various explosives is expressed in terms of the so-called tritol equivalent $\left(k_{T N T}\right)$. If this equivalent cannot be found in the specialized literature (for example [7]), it can be calculated with sufficient accuracy using the relationship

$$
k_{T N T-p}=0.3 Q_{v}-0.2 \quad \text { (for } 2 \mathrm{MJ} / \mathrm{kg} \leq Q_{v} \leq 5 \mathrm{MJ} / \mathrm{kg} \text { ) }
$$

where $k_{T N T-p}$ is the pressure tritol equivalent of the explosive (equal to 1 for TNT), $Q_{v}$ is the calculated explosion heat $[\mathrm{MJ} / \mathrm{kg}]$ and $Q_{v}=4.2 \mathrm{MJ} / \mathrm{kg}$ for TNT. [7]

Then the total equivalent mass $C_{W}$ can be determined using the relationship

$$
C_{w}=C_{N} \cdot k_{T N T-p} \cdot k_{E} \cdot k_{G}
$$

where $C_{w}$ is the mass of the equivalent charge [kg TNT], $C_{N}$ is the mass of the used charge of the (actual) explosive $[\mathrm{kg}], k_{T N T-p}$ is the pressure tritol equivalent, 
$k_{E}$ is the charge seal coefficient, and $k_{G}$ is the geometry coefficient of the impact wave spreading in the space.

The seal coefficient can be determined using the relationship

$$
k_{E}=0.2+0.8 /\left(1+k_{B}\right)
$$

where $k_{B}$ is the cover mass $[\mathrm{kg}]$ divided by the explosive mass $[\mathrm{kg}]$, and expresses the ballistic ratio. The following applies to the geometry coefficient $k_{G}$, 1 for detonation in a free air space, 2 for surface detonation (on the ground).

The explosion wave spreads in spherical wavefronts from the focus point of the explosion. When the explosion is on the ground, the explosion energy is roughly double because, after complete reflection from the surface of the terrain, the pressure wave spreads in hemispherical wavefronts. The spreading geometry coefficient $k_{G}$ is not stated by some authors in the formulas for determining the total equivalent mass; in such cases, and in the case of a ground explosion, the equivalent charge mass $C_{w}$ is as a rule substituted by twice its value in empirical formulas.

In the simplified calculation [7], a ground explosion is represented by a situation when the explosive is located directly on the surface of the terrain $\left(h=0 \mathrm{~m}\right.$ thus $k_{G}=2$ ). An explosion in an open air space is a situation when the delay of the reflected wave from the surface of the terrain to the pressure wave front is higher than the duration of the overpressure phase of the pressure wave $\left(k_{G}=1\right)$. A linear interpolation is made between the values.

On the basis of comparing various resources in the literature (namely $[1,3,4])$ and on the basis of tests of bricked structures $[2,6]$ and window glass [5] during explosions of small charges, the authors of this paper proposed the application of realistic formulas. The empirical formulas below were verified in experiments using small charges (Semtex) in the vicinity of the loaded structure. Their resulting form then corresponds to the impact wave effects from a small solid charge in an external environment and during a ground explosion. Maximum overpressure $p_{+}$and underpressure $p_{-}$at the face of the air impact wave and their durations $\tau_{+}$and $\tau_{-}$are applicable both to ground explosions $\left(C_{W}\right.$ is replaced by the double value of the equivalent charge) and above-ground explosions in a free (air) environment:

$$
\begin{gathered}
p_{+}=\frac{1,07}{\bar{R}^{3}}-0,1 \quad[\mathrm{MPa}] \text { for } \bar{R} \leq 1 \mathrm{~m} / \mathrm{kg}^{1 / 3} \\
p_{+}=\frac{0,0932}{\bar{R}}+\frac{0,383}{\bar{R}^{2}}+\frac{1,275}{\bar{R}^{3}} \quad[\mathrm{MPa}] \text { for } 1<\bar{R} \leq 15 \mathrm{~m} / \mathrm{kg}^{1 / 3} \\
p_{-}=\frac{0,035}{\bar{R}} \quad[\mathrm{MPa}] \\
\tau_{+}=1,6 \cdot 10^{-3} \cdot \sqrt[6]{C_{w}} \cdot \sqrt{R} \quad[\mathrm{~s}]
\end{gathered}
$$




$$
\tau_{-}=1,6 \cdot 10^{-2} \cdot \sqrt[3]{C_{w}} \quad[\mathrm{~s}]
$$

After a normal (perpendicular) impact of the explosion wave on a solid obstacle, a reflected wave is formed with the reflection overpressure $p_{\text {ref }}$ that loads the building structure from the front side (Figure 2). The overpressure value in the reflected wave corresponds to approximately twice the value of the overpressure for low overpressure values $p_{+}$of approximately up to $5 \mathrm{MPa}$ (up to eight times the value for high overpressures of the order of several $\mathrm{MPa}$ ) in the incident wave for the given distance $R[7]$.

$$
\begin{aligned}
& p_{\text {ref }} \approx 2 p_{+} \\
& p_{\text {ref- }} \approx 2 p_{-}
\end{aligned}
$$

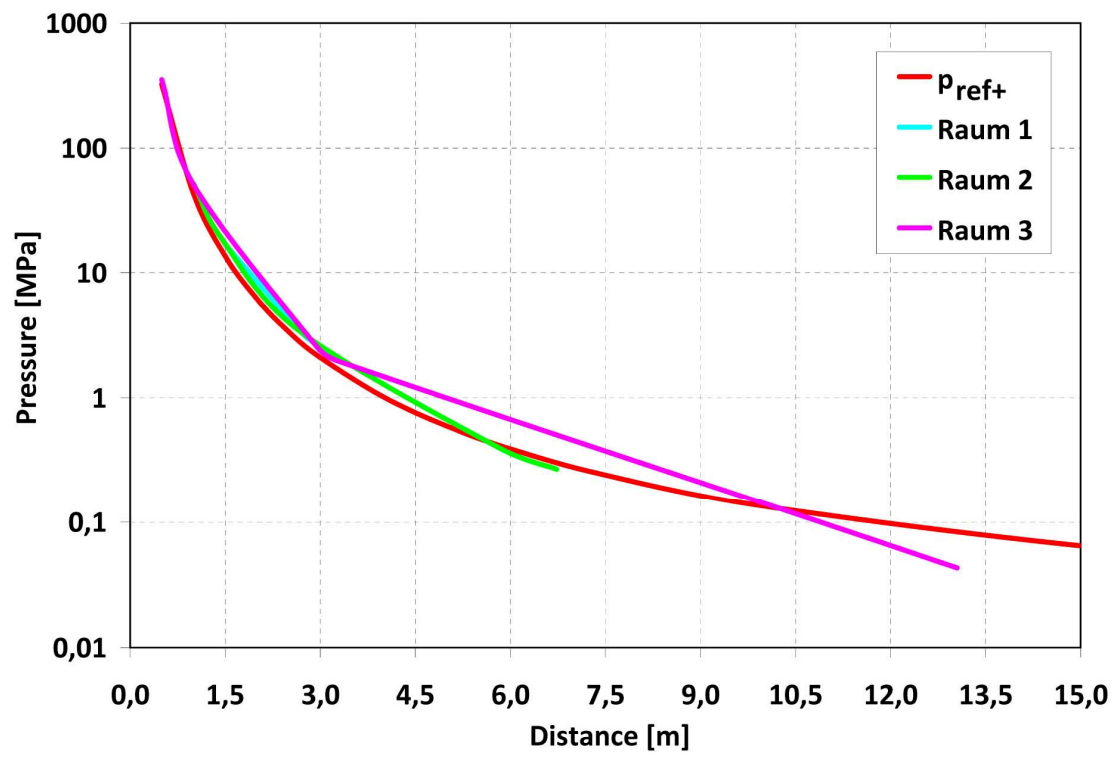

Figure 2: $\quad$ Magnitude of overpressure $p_{\text {ref+ }}$ in dependence on distance $R$ of the charge location compared to the reflection overpressure $p_{\text {ref }}^{f}$ for specific volumes of rooms 1 to 3 .

After an explosion in the enclosed space of rooms in a building structure, with closed relieving vents, the load is increased by approximately $50 \%$ due to reflection from the surface of the walls, the ceiling and the floor of the room; the duration of the overpressure is thus roughly double. The resulting load of the surrounding structures and its duration can be expressed approximately as follows:

$$
\begin{gathered}
p_{\text {load }+} \approx 1.5 \cdot p_{\text {ref }+} \\
t_{\text {load }+} \approx 2 \tau_{+}
\end{gathered}
$$


Formulas similar to those for the overpressure phase of the load also apply approximately to the underpressure phase.

The reflective overpressure $p_{\text {ref }}=p_{\text {ref }}$ in the rooms can also be calculated directly $p_{\text {ref }}=p_{\text {ref }}^{f}$ according to a method described in [9].

To determine the reflective overpressures and impulses, their values in the band $\bar{R}<2 \mathrm{~m} / \mathrm{kg}^{1 / 3}$ must either be read from the published curves [9], or their approximate values must be determined using the derived exponential relationships:

a) Reflective overpressure:

$$
\begin{array}{ccc}
p_{\text {ref }}^{f}=14,554 \times \bar{R}^{-1,4587} & {[\mathrm{MPa}]} & \text { for } 0,05<\bar{R} \leq 0,5 \mathrm{~m} / \mathrm{kg}^{1 / 3} \\
p_{\text {ref }}^{f}=5,76 \times \bar{R}^{-2,762} & {[\mathrm{MPa}]} & \text { for } 0,5<\bar{R} \leq 5 \mathrm{~m} / \mathrm{kg}^{1 / 3}
\end{array}
$$

b) Reflective impulse:

$$
\begin{aligned}
& I_{r e f}^{f}=0,345 \times \sqrt[3]{C_{w}} \times \bar{R}^{-1,857} \quad[\mathrm{kPa} . \mathrm{s}] \quad \text { for } 0,05<\bar{R} \leq 0,5 \mathrm{~m} / \mathrm{kg}^{1 / 3} \\
& I_{\text {ref }}^{f}=0,5823 \times \sqrt[3]{C_{w}} \times \bar{R}^{-1,0976} \quad[\mathrm{kPa} . \mathrm{s}] \quad \text { for } 0,5<\bar{R} \leq 5 \mathrm{~m} / \mathrm{kg}^{1 / 3}
\end{aligned}
$$

When substituting input values $C_{W}$ into the relationships above, two differences from the calculations of the reflective overpressure and the reflective impulse in the open space outside the building must be taken into account:

a) Here, the indices $f$ denote the detonation conditions in the free air space in the room, and the following is substituted for the charge size $C_{W}$ (different from formula (3) above):

b)

$$
C_{w}=C_{N} \cdot k_{T N T-p} \cdot k_{E} \cdot k_{G}=C_{N} \cdot k_{T N T-p} \cdot k_{E}
$$

where the impact wave spreading geometry coefficient $k_{G}=1.0$.

\section{Overpressure calculation for specific rooms}

Figure 2 shows the calculation values of the reflection overpressure $p_{\text {reft }}$ calculated using two different simplified procedures, namely according to formulas (9) to (15) derived for an explosion wave spreading in an external space, and furthermore, in order to compare the results of a direct calculation of the reflective overpressure in the rooms, according to formulas (18) and (19) for three selected rooms according to the methodology in [15]:

Room 1 (Figure 1): Volume $69 \mathrm{~m}^{3}$, exhaust vents $1.7 \mathrm{~m}^{2}$, area of the walls, floor and ceiling $104.9 \mathrm{~m}^{2}$;

Room 2: Volume $69 \mathrm{~m}^{3}$, exhaust vents $1.7 \mathrm{~m}^{2}$, area of the walls, floor and ceiling $91.5 \mathrm{~m}^{2}$; 
Room 3: Volume $255.8 \mathrm{~m}^{3}$, exhaust vents $24.7 \mathrm{~m}^{2}$, area of the walls, floor and ceiling $283.1 \mathrm{~m}^{2}$.

A comparison of the results obtained by the two simplified procedures shows that both methodologies provide sufficient accuracy for the usual volume of medium-size and large rooms, and can be applied to any position in which the charge is deposited in the internal areas of the rooms.

Now the calculated overpressures for selected wall 1 (using the marking based on Figure 1) will be compared, as calculated by the LS DYNA program [10], taking into account the interaction of the room environment with its walls (Fluid-Structure Interaction) in Figure 3. The calculated wall is divided into 16 fields, and the mean overpressure for the suitcase containing the explosive, deposited at the centre of the room, is calculated and shown in each of the fields. This figure shows that, due to reflections from the walls of the room, the explosion peaks are superimposed on each other and their coverage curve roughly corresponds to formulas (16) and (17). The peak pressure coverage is dominant for the global structure response. So that an equivalent "static" calculation is justified for the analysis of this problem.

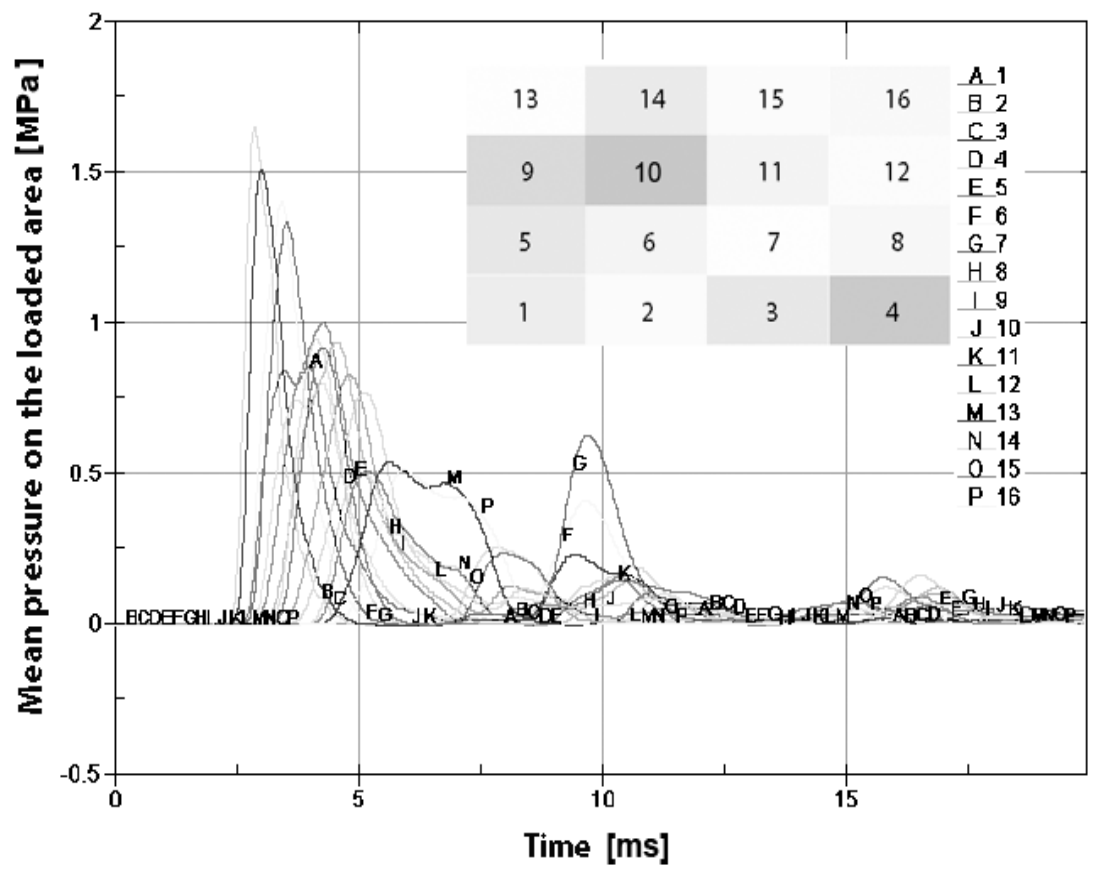

Figure 3: Time course of the mean pressure on the basis of a 3D calculation of the interaction that occurs between the pressure wave from the explosion of a charge in the centre of the room, and the wall at points corresponding to the wall as divided into rectangles. 


\section{Calculating the damage to the walls of the rooms}

In order to evaluate the masonry of the walls and pillars of the room structure, we can use the load estimate $p_{\text {load }+}$ and the duration of its effect $t_{\text {load }+}$, calculated for the possible distance $R$ of the charge position from the evaluated wall, window, door or interwindow or inside pillars.

The equivalent static calculation of the wall uniformly and continuously loaded by load $p_{\text {load }+}$ with duration of its effect $t_{\text {load }+}$ was used to determine the failure hazard. In this procedure, the nature of the boundary conditions (support of the wall board) is chosen. As concerns the partition walls of a specific building, the individual wall board elements were considered to be independent, for the sake of simplification, hinge-mounted along their entire circumference.

From the viewpoint of uncertainty in the simplified effect of the pressure wave from the explosion, even such a simplification is acceptable and justified for an engineering estimate of the explosion effects.

As a rule, the dynamic coefficient $\delta$ is derived for the equivalent static calculation, and for a system with one degree of freedom it is the function of the natural period of dominant oscillation $T$ of the structure and the pressure wave effect duration $\tau_{+}$or $\tau_{-}$, based on whether the overpressure or underpressure wave is considered.

For the elastic-plastic system, dynamic coefficient $\delta$ is the function of the ratio of the impact wave effect duration $\tau_{+}$or $\tau_{-}$on the natural structure oscillation period $T_{(i)}=T$ and on the ductility of the structure:

$$
k_{m}=\frac{y_{m}}{y_{e l}}
$$

where $y_{m}$ is the total elastic + plastic deflection (shift) of the structure, and $y_{e l}$ is the elastic part of the deflection (shift).

As for impact phenomena (very rapid) during bending stress of the structure, the ductility coefficient $k_{m}$ can usually be considered to be equal to 3 to 5 for masonry, and from 5 to 10 for reinforced concrete, steel and wood. As for the load due to the impact wave, the dynamic coefficient including consideration of the ductile behaviour of the structure is found to be in the range $\delta=1 \sim 2$. This magnitude was derived by N. M. Newmark (see [3]) for a simplified system with one degree of freedom in the following form:

$$
\frac{1}{\delta}=\frac{T_{(i)} \cdot \sqrt{2 \cdot k_{m}-1}}{\pi \cdot \tau_{+}}+\frac{1-\frac{1}{2 \cdot k_{m}}}{1+0,7 \cdot \frac{T_{(i)}}{\tau_{+}}}
$$

\section{Evaluating the failure probability for a specific room}

When calculating the load level $p_{\text {load }+}$, the load is found to be in the range of units of $\mathrm{MPa}$ or hundreds of $\mathrm{kPa}$ inside a room (Figure 1), based on the position 
of the charge inside the room. When comparing such high loads with the carrying capacity of the windows and doors, it reaches several units of $\mathrm{kPa}$. It is apparent that such window and door openings will be smashed (destroyed) and will enable the pressure to be released into the surrounding (external or internal) areas.

Table 1 shows the calculated bending moments in the middle part of the wall board in vertical and horizontal directions, maximum deflection $y$ at the centre of the wall board and the angle $\varphi$ of angular displacement of the centre line of the wall board. The angle of $5^{\circ}$ was chosen as the limit angle $\psi$ at which the wall board masonry breakdown occurs (fracture, sweeping out of brick fragments, etc.). For the sake of transparency, the individual walls of the room are

Table 1: $\quad$ Failure risk estimation of structural parts (Figure 1).

\begin{tabular}{|c|c|c|c|c|c|c|c|c|}
\hline \multirow{3}{*}{ 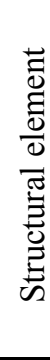 } & 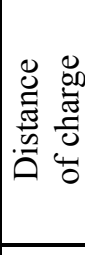 & 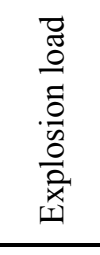 & 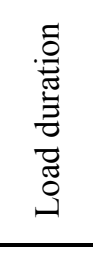 & 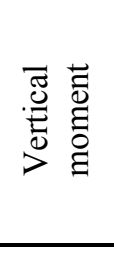 & 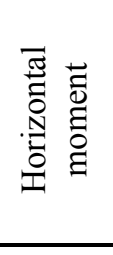 & 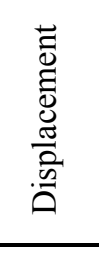 & 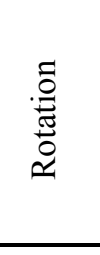 & \multirow{3}{*}{ 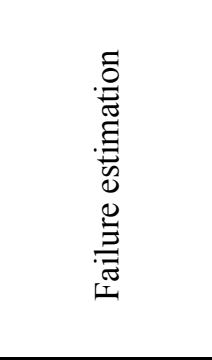 } \\
\hline & $R$ & $p_{\text {load }+}$ & $t_{\text {load }+}$ & $M_{v e r}$ & $M_{\text {hor }}$ & $y$ & $\varphi$ & \\
\hline & {$[\mathrm{m}]$} & [MPa] & {$[\mathrm{s}]$} & {$[\mathrm{kNm}]$} & {$[\mathrm{kNm}]$} & {$[\mathrm{mm}]$} & [deg] & \\
\hline \multirow{3}{*}{1} & \multicolumn{8}{|c|}{ Wall $6700 \times 2800 \times 100 \mathrm{~mm}$} \\
\hline & 1.5 & 20.53 & 0.006 & 360 & 141 & 7088 & 157.7 & Expected \\
\hline & 2.5 & 5.09 & 0.008 & 116 & 45 & 2282 & 117.0 & Expected \\
\hline \multirow{3}{*}{2} & \multicolumn{8}{|c|}{ Wall $5430 \times 2800 \times 100 \mathrm{~mm}$} \\
\hline & 2 & 9.27 & 0.007 & 188 & 76 & 3697 & 138.5 & Expected \\
\hline & 5 & 0.89 & 0.011 & 28 & 11 & 557 & 43.4 & Expected \\
\hline \multirow{4}{*}{3} & \multicolumn{8}{|c|}{ Wall $6700 \times 2800 \times 520 \mathrm{~mm}$} \\
\hline & 1 & 64.73 & 0.005 & 4902 & 1924 & 688 & 52.3 & Expected \\
\hline & 3 & 3.15 & 0.009 & 409 & 160 & 57 & 4.7 & Partial failure \\
\hline & 6 & 0.58 & 0.012 & 105 & 41 & 15 & 1.2 & Improbable \\
\hline \multirow{4}{*}{4} & \multicolumn{8}{|c|}{ Wall $5430 \times 2800 \times 1100 \mathrm{~mm}$} \\
\hline & 1 & 64.74 & 0.005 & 10202 & 4122 & 151 & 12.3 & Probable \\
\hline & 2 & 9.27 & 0.007 & 2030 & 820 & 30 & 2.5 & Improbable \\
\hline & 4 & 1.52 & 0.010 & 460 & 186 & 7 & 0.6 & Improbable \\
\hline \multirow{4}{*}{5} & \multicolumn{8}{|c|}{ Pillar $1630 \times 2800 \times 1100 \mathrm{~mm}$} \\
\hline & 1 & 64.74 & 0.005 & 9227 & 4050 & 44 & 6.3 & Partial failure \\
\hline & 2 & 9.27 & 0.007 & 1751 & 769 & 8 & 1.2 & Improbable \\
\hline & 4 & 1.52 & 0.010 & 369 & 162 & 2 & 0.3 & Improbable \\
\hline
\end{tabular}


numbered and these numbers are shown in the ground plan of the room in Figure 1. It follows clearly from Table 1 that thin partition walls up to $150 \mathrm{~mm}$ in thickness of will be destroyed by the explosion.

As the explosion pressures markedly exceed the carrying capacity of such thin partition walls, the ruins of the partition walls will be swept into the surrounding areas. Thick bricked walls and interwindow pillars $900 \mathrm{~mm}$ and more in thickness will be destroyed only if the charge is placed in their vicinity, at a distance of about $1 \mathrm{~m}$. For distances of the charge of more than $2 \mathrm{~m}$, such a massive structure will transfer the explosion pressures without collapsing and without any other serious defects. Of course, the plaster will be damaged, cracks will appear in the walls, brick fragments may fall out, etc., but the structure will not collapse.

If a massive carrying wall or pillar (more than $900 \mathrm{~mm}$ in thickness) collapses under this ceiling, it is likely that the ceiling structure will fall through and damage will also occur to higher floors.

\section{Conclusions}

An example of a specific building was used to discuss the explosion and the building safety hazard when a terrorist charge is brought into the building in a suitcase and is equipped with a system for initiating the charge after it has been placed in the building and the terrorist has left.

Due to uncertainties in all parameters of the explosion load, a simplified methodology has been presented here. This methodology enables the parameters to be determined sufficiently concisely and the natural building structure to be evaluated on the basis of the parameters. The uncertainty in determining the explosion load parameters can be determined on the basis the results of a calculation using empirical formulas derived by the authors for small charges. The response of the structure is evaluated on the basis of the results of the equivalent static calculation, using the dynamic coefficient for the elastic-plastic system. The explosion hazard of the structure is evaluated on the basis of the maximum moments and deflections of the structure.

The example of a specific room is used to analyze its exposure and also the hazard to the entire building based on various possible placements of a charge a short distance or a longer distance away from the carrying structure and the partition walls.

\section{Acknowledgement}

This research was supported as a part of the research projects in GAČR 103/08/0859 "Structure response under static and dynamic loads caused by natural and man induced activity", for which the authors would like to thank the Agency. 


\section{References}

[1] Henrych, J., The Dynamics of Explosion and Its Use, Academia: Prague 1979.

[2] Janovský, B., Šelešovský, P., Horkel, J. \& Vejs, L., Vented Confined Explosions in Stramberk Experimental Mine and AutoReaGas Simulation. J. Loss Prevention in the Process Industries, Vol. 19, pp. 280-287, 2006.

[3] Koloušek, V. et al., Building Structures Under Dynamic Effects (in Slovak), SVTL: Bratislava 1967.

[4] Korenev, B.G. et al., Dynamic Calculation of Structures Under Special Effects (in Russian), Strojizdat: Moskva 1981.

[5] Makovička, D., Shock Wave Load of Window Glass Plate Structure and Hypothesis of Its Failure. In: Structures Under Shock and Impact '98, pp. 43-52, WIT Press: Southampton 1998.

[6] Makovička, D., Failure of Masonry Under Impact Load Generated by an Explosion. Acta Polytechnica, Vol. 39, No. 1/1999, pp. 63-91.

[7] Makovička, D. \& Janovský, B., Handbook of Explosion Protection for Buildings (in Czech), CTU Publishing House in Prague, 2008.

[8] Makovička, D., Makovička, D., Janovský, B. \& Adamík, V., Exposure of building structure to charge explosion in interior (in Czech), Stavebni obzor, Vol. 18, No. 9/2009, pp. 257-265.

[9] Baker, W.E., Westine, P.S., Cox, P.A. et al., Explosion Hazards and Evaluation, Elsevier: Amsterdam 1983.

[10] LS-DYNA User's Manual, Nonlinear Dynamic Analysis of Structures, Version 950, Livermore Software Technology Corporation, May 1999. 